\title{
A single institution analysis of recurrence in myxoid liposarcoma
}

\author{
Dean Wang ${ }^{1}$, Karen J. Fritchie ${ }^{2}$, Amy S. Nowacki ${ }^{3}$, Brian P. Rubin ${ }^{2}$, Steven Lietman ${ }^{4}$ \\ 1. Department of Orthopaedic Surgery, David Geffen School of Medicine, University of California Los Angeles, 10833 Le \\ Conte Avenue, Room 76-143, Los Angeles, CA, 90095, United States. 2. Department of Anatomic Pathology, Cleveland \\ Clinic Foundation, 9500 Euclid Avenue, L25, Cleveland, OH 44195, United States. 3. Department of Quantitative Health \\ Sciences, Cleveland Clinic Desk JJ N3-01, 9500 Euclid Avenue, Cleveland, OH 44195, United States. 4. Department of \\ Orthopaedic Surgery, Cleveland Clinic Foundation, 9500 Euclid Avenue, A41, Cleveland, OH 44195, United States.
}

Correspondence: Steven A. Lietman. Address: Department of Orthopaedic Surgery, Cleveland Clinic Foundation, 9500 Euclid Avenue, A41, Cleveland, OH 44195, United States. Email: lietmas@ccf.org

Received: May 26, 2013

DOI : $10.5430 /$ jst.v3n4p44

Accepted: August 19, 2013

Online Published: August 21, 2013

\section{Abstract}

Background: Myxoid liposarcomas account for approximately one third of all liposarcomas and typically present as a slow-growing, deep-seated tumor in the lower extremity. Although approximately one-third of patients develop metastases and eventually die from their tumors, prognostic factors for recurrence have not been thoroughly studied. The effects of radiotherapy and perioperative allogeneic blood transfusions on tumor recurrence in primary myxoid liposarcomas were investigated.

Patients and Methods: A retrospective review of 47 primary myxoid liposarcomas treated at a single institution was completed. Morphologic diagnoses were confirmed by soft tissue pathologists without prior knowledge of the clinical features, treatment, or outcome. Associations among treatment factors and recurrence were examined.

Results: Four (9\%) and fourteen (30\%) patients developed local recurrences and distant metastases, respectively. The median follow-up time was 54 months. There were no local recurrences among those who received radiotherapy, thus producing a significant decrease in local recurrence in those patients who received radiotherapy ( $p[\mathrm{Cox}$ model $]=0.010$ ). The receipt of $\geq 3$ blood transfusions correlated with an increase in tumor local recurrence ( $p[$ Cox model $]=0.030$ ).

Conclusions: Radiotherapy and perioperative blood transfusion quantity may have an effect on tumor recurrence and warrant further investigation.

Mini-Abstract: Radiotherapy and perioperative blood transfusion quantity have a significant effect on myxoid liposarcoma recurrence.

\section{Key words}

Sarcoma, Cancer, Blood transfusion, Radiotherapy

\section{I ntroduction}

Liposarcomas are malignant soft tissue tumors that arise from primitive mesenchymal cells and express adipocytic features. Four major subtypes of liposarcoma are recognized: well-differentiated, myxoid (myxoid/round cell), 
pleomorphic, and mixed ${ }^{[19]}$. Myxoid liposarcomas account for approximately one third of all liposarcomas and typically present as slow-growing, deep-seated tumors in the lower extremity ${ }^{[4]}$. Once considered a separate entity, round cell liposarcomas are now considered in the same spectrum as myxoid liposarcomas, sharing a fusion of the TLS and CHOP genes, $\mathrm{t}(12 ; 16)(\mathrm{q} 13-14 ; \mathrm{p} 11)$ in more than $90 \%$ of cases ${ }^{[16]}$.

Previous reports have shown that myxoid liposarcomas with a $\geq 5 \%$ round cell component are at increased risk for death due to disease ${ }^{[4,23,25]}$. However, predictors of tumor recurrence risk after surgical excision are less well defined. Positive surgical margins are common due to large and invasive nature of myxoid liposarcomas and correlate with increased local recurrence rates ${ }^{[1,15,17]}$. Despite a significant percentage of positive margins, limb salvage is still thought to be possible with radiotherapy, providing an adjunct for effective local control. However, the success of radiotherapy in these tumors has not been well established. Some studies have reported higher local recurrence rates (24-33\%) than others, and this may be attributed to the less frequent use of radiotherapy in their cohorts ${ }^{[1,8,25]}$.

Risk factors for the metastasis of myxoid liposarcomas are also less well defined. Approximately one-third of myxoid liposarcoma patients develop metastases and eventually die from their tumors ${ }^{[11,20]}$. Tumor size and $\geq 5 \%$ round cell component are significantly associated with the development of metastasis ${ }^{[6,8,15,17]}$. The effect of perioperative blood transfusion on metastasis is more controversial. Some argue that it is immunosuppressive due to leukocytes and leukocyte byproducts present in stored blood and may increase the risk of recurrence in soft tissue sarcoma patients ${ }^{[17,21]}$, while others debate the matter ${ }^{[13]}$.

The aims of this investigation of primary myxoid liposarcomas were to determine if (1) radiotherapy improves local control; and (2) the receipt of perioperative allogeneic blood transfusions increases tumor recurrence.

\section{Patients and methods}

\subsection{Patient selection and data collection}

Between January 1, 1980 and May 30, 2010, 47 patients underwent surgical resection for primary myxoid liposarcoma of the extremity at our institution. Original tumor blocks and slides were collected from the Anatomic Pathology database at our institution. The morphologic diagnosis for 39 tumors was confirmed by at least one pathologist (K.F. or B.P.R.) with expertise in soft tissue pathology without prior knowledge of the clinical features, treatment, or outcome. Criteria for diagnosis of myxoid liposarcoma and round cell component are detailed by Fritchie et al ${ }^{[9]}$. Eight other tumors received neoadjuvant treatment, and the diagnoses from these pre-treatment biopsies were used because the morphologic diagnoses post-resection could not be confirmed. Approval for use of human data was obtained from the IRB 08-377 at our institution for this investigation.

Data was collected retrospectively on the 47 patients via review of the electronic medical records and paper records. Patient characteristics collected included age at time of surgery, gender, and smoking history. Smoking status was categorized by any past smoking history before initial presentation. Tumor features collected included primary site, dimensional size, margin status and measured margin length, and tissue type adjacent to the smallest margin. An attempt to perform a complete resection with negative margins was performed in all patients. Margins were defined at the time of pathologic assessment by a dedicated pathologist, who evaluated specimens both grossly and microscopically in six dimensions (superior, inferior, medial, lateral, anterior, and posterior). Negative margins indicated that there was no discernible tumor at the edge of an inked specimen. Several tumors that were within $<1 \mathrm{~mm}$ of the edge but were enveloped by a fibrous capsule were still considered to have negative margins. A positive margin was determined by gross or microscopic discernible tumor extension to the edge of an inked specimen. Adjuvant treatment data was gathered for the preoperative, perioperative, and postoperative time periods. Complete transfusion records were available from the Blood 
Bank, which began recording data in July 1998, for 23 of the 47 verified tumors. All patients who underwent a blood transfusion within 30 days of the index surgery were considered as having received a perioperative transfusion.

Outcome endpoints collected include date of documented first local recurrence, date of documented first distant metastasis, date of last follow-up, and if applicable, date of death. Time to event was calculated from the date of the index surgery to the date of documented discovery. Date of last follow-up was recorded based on the patients' last office visit with a member of the oncology team (surgeon, medical oncologist, or radiation oncologist). Surveillance for tumor recurrence was generally completed during follow-up clinical examinations three months after the index surgery, every three to four months for two years, and then every six months for up to five years. Annual clinical examinations and radiographs were performed thereafter. For patients with a disease-free survival of more than five years and no recent follow-up, the Cleveland Clinic Tumor Registry made annual phone calls to the patient or patient's family to ascertain death or any evidence of disease. Additionally, the U.S. Social Security Death Index was queried to confirm patient survival. For deceased patients, determination of the cause of the death was through review of the medical record.

\subsection{Statistical methods}

All outcome analyses were completed using the Kaplan-Meier method, and curves were compared by using the log-rank test. Associations with events of interest were verified with a competing risks analysis using death as the competing risk. Outcome analyses were also completed using the Cox proportional hazards likelihood ratio test via bivariable associations. Comparison between rates and proportions were assessed with chi-square analysis or the Fisher exact test as appropriate. Differences were considered significant for $p<0.05$. JMP 9.0 software (SAS Institute, Cary, NC) was used for all analyses and graphs.

\section{Results}

After pathologic review, data for 47 primary myxoid liposarcomas was collected (Table 1). The median follow-up time was 54 months for all patients and 60 months for survivors. Thirty-two patients (68\%) remain alive at last follow-up. All patients were evaluated by a medical oncologist and radiation oncologist and were selected for adjuvant therapies based on presence of round cell component, positive margins, and/or size $>10 \mathrm{~cm}$. Due to the large time span of this study, no uniform selection criteria could be ascertained for the receipt of adjuvant therapies or blood transfusions. The 5- and 12-year overall survival rates were 68\% (95\% CI, 52\%-81\%) and 57\% (95\% CI, 37\%-75\%), respectively (Figure 1).

There were no local recurrences among those who received radiotherapy, thus producing a significant difference in recurrence incidence between those who received radiotherapy and those who did not $(p[\log$-rank $]=0.022, p[\mathrm{Cox}$ model $]=0.010$ ) (Figure 2). This association remained after treating death as a competing risk $(p=0.025)$. A total of 26 patients $(55 \%)$ received radiotherapy ( 5 preoperative, 1 perioperative, 17 postoperative) and three patients received multiple phases of radiotherapy. Seven patients (15\%) received both radiotherapy and chemotherapy. All patients with positive surgical margins received radiotherapy. Four out of 47 total patients $(9 \%)$ developed a local recurrence. Each of these 4 patients with local recurrence did not receive radiation and the median time to recurrence was 24 months. Three of these four patients remained alive at last follow-up, resulting in a median survival time after event of 51 months. Interestingly, three of these four patients also experienced a distant metastasis of their tumor. One of these four patients developed metastasis to the lung 17 months before recurring locally.

In the 23 patients for whom blood transfusion data was available, the differences in local recurrence and distant metastatic rates between those who did and did not receive a perioperative allogeneic blood transfusion did not reach statistical significance ( $p=0.146, p=0.181$, respectively). However, among those who received a transfusion of $\geq 3$ units, a trend towards increased local recurrence was noted by $p[\log$-rank $]=0.059$, and a significant difference was noted by $p[$ Cox model $]=0.030$ (Table 2 ). This trend remained after treating death as a competing risk $(p=0.072)$. Fourteen patients received allogeneic packed red blood cell transfusions (12 leukoreduced, 2 non-leukoreduced). Two of the patients receiving 
allogeneic packed red blood cells also received pooled platelets and fresh frozen plasma. Patients were categorized as receiving either $\geq 3$ units (8), 1-2 units (6), or no (9) allogeneic packed red blood cells. Three of the 8 patients who received $\geq 3$ units had a local recurrence, and there were no local recurrences among the 6 patients who received 1-3 units. Blood transfusions did not lead to an increase in metastasis (Table 3).

Table 1. Clinicopathologic and treatment characteristics of 47 patients with primary myxoid liposarcoma

\begin{tabular}{|c|c|c|}
\hline Patient Characteristic & $\mathbf{n}$ & $\%$ of Total \\
\hline Age (yr) [median (range)] & $50(23-81)$ & \\
\hline \multicolumn{3}{|l|}{ Gender } \\
\hline Male & 27 & 57.4 \\
\hline Female & 20 & 42.6 \\
\hline \multicolumn{3}{|l|}{ Smoking history } \\
\hline Yes & 25 & 53.2 \\
\hline No & 22 & 46.8 \\
\hline \multicolumn{3}{|l|}{ Histotype } \\
\hline$<5 \%$ RC Component & 16 & 34.0 \\
\hline$\geq 5 \%$ RC Component & 16 & 34.0 \\
\hline \multicolumn{3}{|l|}{ Primary site } \\
\hline Lower extremity & 45 & 95.7 \\
\hline Upper extremity & 2 & 4.3 \\
\hline Tumor diameter $(\mathrm{cm})$ [median (range)] & $15(0.6-45)$ & \\
\hline Tumor volume (cc) [median(range) $]^{*}$ & $3,638(17-42,412)$ & \\
\hline \multicolumn{3}{|l|}{ Surgical margin } \\
\hline Positive & 7 & 14.9 \\
\hline Negative & 40 & 85.1 \\
\hline \multicolumn{3}{|l|}{ Transfusion $^{\#}$} \\
\hline$>3 \mathrm{U}$ & 8 & 34.8 \\
\hline $1-3 U$ & 6 & 26.1 \\
\hline No & 9 & 39.1 \\
\hline \multicolumn{3}{|l|}{ Radiation therapy } \\
\hline Yes & 26 & 55.3 \\
\hline No & 21 & 44.7 \\
\hline \multicolumn{3}{|l|}{ Chemotherapy } \\
\hline Yes & 11 & 23.4 \\
\hline No & 36 & 76.6 \\
\hline
\end{tabular}

${ }^{*} 42$ patients, ${ }^{*} 23$ patients

\section{Discussion}

Myxoid liposarcomas are intermediate risk tumors that molecularly share a fusion of the TLS and CHOP genes. Previous studies that investigate prognostic factors for liposarcomas group myxoid/round cell subtypes with all other histologic 
subtypes in their analyses. However, each liposarcoma subtype is vastly different in terms of their risks of recurrence and death, and thus, investigations should focus on each separately to more accurately characterize clinical predictors. The present investigation of primary myxoid liposarcomas asked if (1) radiotherapy improves local control and if (2) the receipt of perioperative allogeneic blood transfusions increases local tumor recurrence.

Table 2. Summary of patient, tumor, treatment and transfusion factors by local recurrence

\begin{tabular}{llll}
\hline Factor & $\begin{array}{l}\text { Local Recurrence } \\
(\mathbf{n}=\mathbf{4})\end{array}$ & $\begin{array}{l}\text { No Local Recurrence } \\
(\mathbf{n}=\mathbf{4 3})\end{array}$ & Cox proportional model $\boldsymbol{p}$ value \\
\hline Age & $49.5(15.0)$ & $49.8(12.1)$ & 0.972 \\
Male & $1(25 \%)$ & $26(60 \%)$ & 0.144 \\
Positive smoking history & $1(25 \%)$ & $24(56 \%)$ & 0.168 \\
Tumor diameter & $14.9(10.2)$ & $14.4(8.2)$ & 0.631 \\
Tumor volume & $15745(3607)$ & $6067(8803)$ & 0.101 \\
Surgical margin positive & $0(0 \%)$ & $7(16 \%)$ & 0.266 \\
Received transfusion & $3(100 \%)$ & $11(55 \%)$ & 0.074 \\
Transfusion of $\geq 3$ units ${ }^{\#}$ & $3(100 \%)$ & $5(45 \%)$ & 0.030 \\
Received radiation therapy & $0(0 \%)$ & $26(60 \%)$ & 0.010 \\
Received chemotherapy & $1(25 \%)$ & $10(23 \%)$ & 0.752 \\
\hline
\end{tabular}

mean (standard deviation) for continuous variables; count (percent) for categorical variables

*Local recurrence, $\mathrm{n}=3$; No local recurrence, $\mathrm{n}=20$

${ }^{\#}$ Local recurrence, $\mathrm{n}=3$; No local recurrence, $\mathrm{n}=11$

Table 3. Summary of patient, tumor, treatment and transfusion factors by distant metastasis

\begin{tabular}{llll}
\hline Factor & $\begin{array}{l}\text { Distant Metastasis } \\
(\mathbf{n}=\mathbf{1 4})\end{array}$ & $\begin{array}{l}\text { No Distant Metastasis } \\
(\mathbf{n}=\mathbf{3 3})\end{array}$ & Cox proportional model p value \\
\hline Age & $49.6(10.1)$ & $49.8(13.1)$ & 0.840 \\
Male & $8(57 \%)$ & $19(58 \%)$ & 0.896 \\
Positive smoking history & $8(57 \%)$ & $17(52 \%)$ & 0.830 \\
Tumor diameter & $16.2(9.0)$ & $13.8(7.9)$ & 0.116 \\
Tumor volume & $8615(9813)$ & $5592(8402)$ & 0.046 \\
Surgical margin positive & $2(14 \%)$ & $5(15 \%)$ & 0.910 \\
Received transfusion ${ }^{*}$ & $5(83 \%)$ & $9(53 \%)$ & 0.157 \\
Transfusion of $\geq 3$ units ${ }^{\#}$ & $3(60 \%)$ & $5(56 \%)$ & 0.692 \\
Received radiation therapy & $8(57 \%)$ & $18(55 \%)$ & 0.968 \\
Received chemotherapy & $5(36 \%)$ & $6(18 \%)$ & 0.087 \\
\hline
\end{tabular}

mean (standard deviation) for continuous variables; count (percent) for categorical variables

*Distant metastasis, $\mathrm{n}=6$; No distant metastasis, $\mathrm{n}=17$

"Distant metastasis, $\mathrm{n}=5$; No distant metastasis, $\mathrm{n}=9$

This review was limited by several factors. Because there were few local recurrences or metastases, no definitive conclusions could be made regarding the effects of radiotherapy and perioperative blood transfusions. Additionally, as is typical with this kind of study, patients receiving neoadjuvant or adjuvant therapies likely had higher-risk disease, introducing a selection bias. Third, although most tumors underwent central review by sarcoma pathologists, the eight 
pre-treated tumors had suboptimal viabilities at the time of resection. Therefore, only the biopsy specimens could be used for the diagnosis confirmation. Fourth, the large time span for the cases most likely led to a degree of variation in the management of these tumors, as there was no consistency in the indications for adjuvant therapies or blood transfusions. Finally, survival and time to event analysis was somewhat limited by the number of patients who had short-term follow-up or were lost over time.

Figure 1. Overall Survival for Myxoid Liposarcoma. Kaplan-Meier curve with overall survival as a function of time in years for patients with myxoid liposarcoma

Figure 2. Local Recurrence for Myxoid Liposarcoma with and without radiation therapy. Probability of local recurrence as a function of time in years. Gray's test $p$-value $=0.025$, Log-rank test $p$-value $=0.022$
Kaplan-Meier Curve for Myxoid Liposarcomas Study

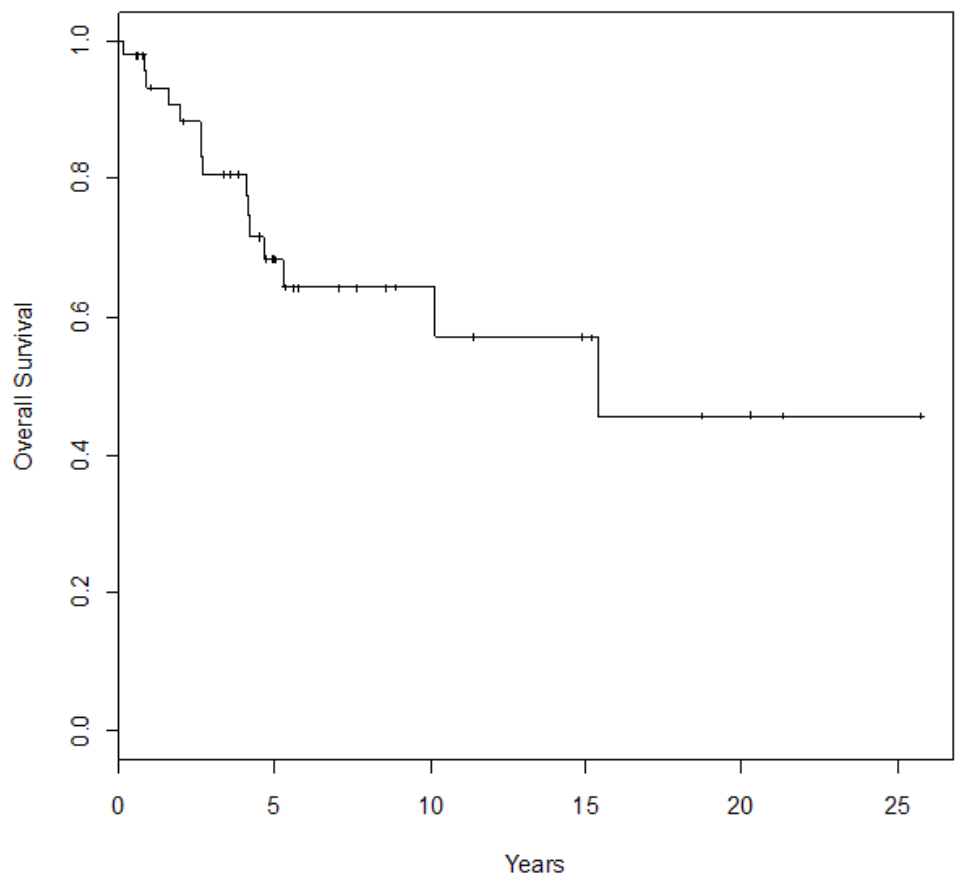

Myxoid liposarcomas dataset: CIFs for local recurrence

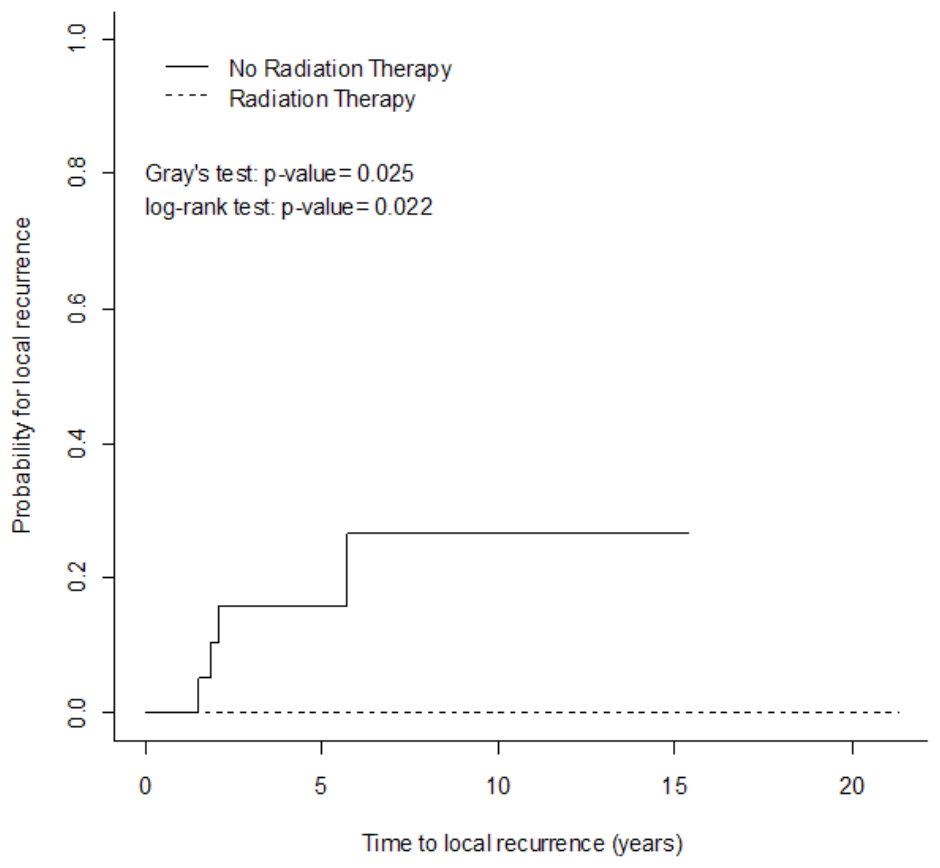


In this series in which more than half of patients (55\%) received neoadjuvant or adjuvant radiotherapy, the risk of local recurrence was $8 \%$ at 5 years, which compares favorably with other reported series in which half or more of patients received postoperative radiotherapy ${ }^{[8,12]}$. The lowest rate of local recurrence in patients with myxoid liposarcoma has been reported from the MD Anderson Cancer Center, in which only 3\% of 127 patients (all of whom were treated with surgery and neoadjuvant and/or adjuvant radiotherapy) developed a local recurrence ${ }^{[10]}$. Perhaps the strongest argument for the effect of radiotherapy providing local control comes from the large Canadian multicenter study consisting of 415 cases, in which the addition of radiotherapy significantly prevented local relapse (18\% vs. $4 \%$ ) and also reduced by sevenfold the 5-year local recurrence rate in patients with positive margins ${ }^{[17]}$. Despite the slow-growing, paucicellular nature of myxoid liposarcoma, radiotherapy has been shown to significantly reduce tumor volume and dramatically change the morphology of myxoid liposarcoma in comparison to malignant fibrous histiocytomas ${ }^{[5,21]}$. Thus, the current collection of findings supports the use of radiotherapy in the neoadjuvant setting and for those with positive margins.

The influence of perioperative blood transfusions on tumor recurrence is more controversial. The source of immunosuppression in patients who are transfused comes from the leukocytes and leukocyte byproducts present in stored blood. Whether perioperative blood transfusions and the subsequent immunological suppression would accelerate recurrent tumor growth and the appearance of metastatic disease is a matter of debate. The strongest evidence for immunosuppression and subsequent tumor growth and the singular role of leukocytes in mediating this effect comes from animal studies ${ }^{[2,3]}$. However, both observational studies and randomized control studies have yielded conflicting results ${ }^{[13,18,22]}$. Additionally, due to the universal adoption of leukoreduction in the last decade, any immunomodulatory effect that transfusions introduce, if real, may be diluted and thus challenging to study. The present study noted a difference in local recurrence in patients who received $\geq 3$ units of non-leukoreduced blood, but there were too few patients to label non-leukoreduced transfusion as a predictor for local recurrence. Of note, two patients in this study received non-leukoreduced blood, and these two eventually experienced an adverse event.

Interestingly, three out of four patients in this series with a local recurrence also developed a distant metastasis. In another series, $68 \%$ of patients who had a local recurrence developed metastases ${ }^{[12]}$. Whether the local recurrence represents a marker of an aggressive tumor or there is a causal association between local recurrence and metastasis is unknown. Some metastases in this patient group were observed in unusual soft tissue locations, such as the retroperitoneum and chest wall, which is a feature of myxoid liposarcoma that has been well described ${ }^{[7,8,24]}$. There was a trend towards increased distant metastasis in patients who received adjuvant chemotherapy, and this may be due to a selection bias towards patients with high-risk disease. Anecdotally, myxoid liposarcomas are chemo-resistant, although some reports have found them to be relatively responsive to doxorubicin-, dacarbazine-, and ifosfamide-based chemotherapy in the metastatic and neoadjuvant setting ${ }^{[14,20]}$. The effectiveness of chemotherapy in the adjuvant setting remains unknown.

Despite the low number of events, this series is large for a single institution and for a single diagnosis of myxoid liposarcoma, and the median follow-up period compares favorably with those reported in the literature. The data suggests that radiotherapy and the quantity of perioperative blood transfusions (or more specifically, the quantity of transfused donor leukocytes and byproducts) may affect the local recurrence of myxoid liposarcomas.

\section{Ethical review committee statement}

Approval regarding use of human data was obtained from the Cleveland Clinic IRB 08-377 for this investigation.

\section{Conflict of interest statement}

There was no external funding source for this investigation. Each author certifies that he or she has no commercial associations (e.g., consultancies, stock ownership, equity interest, patent/licensing arrangements, etc.) that might pose a conflict of interest in connection with this investigation. 


\section{References}

[1] Antonescu CR, Tschernyavsky SJ, Decuseara R, et al. Prognostic impact of P53 status, TLS-CHOP fusion transcript structure, and histological grade in myxoid liposarcoma: a molecular and clinicopathologic study of 82 cases. Clin Cancer Res. 2001; 7(12): 3977-3987. PMid:11751490

[2] Blajchman MA, Bardossy L, Carmen R, Sastry A, Singal DP. Allogeneic blood transfusion-induced enhancement of tumor growth: two animal models showing amelioration by leukodepletion and passive transfer using spleen cells. Blood. 1993; 81(7): 1880-1882. PMid:8461473

[3] Bordin JO, Bardossy L, Blajchman MA. Growth enhancement of established tumors by allogeneic blood transfusion in experimental animals and its amelioration by leukodepletion: the importance of the timing of the leukodepletion. Blood. 1994; 84(1): 344-348. PMid:8018929

[4] Dalal KM, Kattan MW, Antonescu CR, Brennan MF, Singer S. Subtype specific prognostic nomogram for patients with primary liposarcoma of the retroperitoneum, extremity, or trunk. Ann Surg. 2006; 244(3): 381-391. PMid:16926564

[5] Engstrom K, Bergh P, Cederlund CG, et al. Irradiation of myxoid/round cell liposarcoma induces volume reduction and lipoma-like morphology. Acta Oncol. 2007; 46(6): 838-845. PMid:17653909 http://dx.doi.org/10.1080/02841860601080415

[6] Engstrom K, Bergh P, Gustafson P, et al. Liposarcoma: outcome based on the Scandinavian Sarcoma Group register. Cancer. 2008; 113(7): 1649-1656. PMid:18720363 http://dx.doi.org/10.1002/cncr.23784

[7] Estourgie SH, Nielsen GP, Ott MJ. Metastatic patterns of extremity myxoid liposarcoma and their outcome. J Surg Oncol. 2002; 80(2): 89-93. PMid:12173385 http://dx.doi.org/10.1002/jso.10093

[8] Fiore M, Grosso F, Lo Vullo S, et al. Myxoid/round cell and pleomorphic liposarcomas: prognostic factors and survival in a series of patients treated at a single institution. Cancer. 2007; 109(12): 2522-2531. PMid:17510918 http://dx.doi.org/10.1002/cncr.22720

[9] Fritchie KJ, Goldblum JR, Tubbs RR, et al. The expanded histologic spectrum of myxoid liposarcoma with an emphasis on newly described patterns: implications for diagnosis on small biopsy specimens. American journal of clinical pathology. 2012; 137(2): 229-239. PMid:22261448 http://dx.doi.org/10.1309/AJCP90YNOKBAGCDM

[10] Guadagnolo BA, Zagars GK, Ballo MT, et al. Excellent local control rates and distinctive patterns of failure in myxoid liposarcoma treated with conservation surgery and radiotherapy. International journal of radiation oncology, biology, physics. 2008; 70(3): 760-765. PMid:17892916 http://dx.doi.org/10.1016/j.ijrobp.2007.07.2337

[11] Gustafson P, Rydholm A, Willen H, Baldetorp B, Ferno M, Akerman M. Liposarcoma: a population-based epidemiologic and prognostic study of features of 43 patients, including tumor DNA content. International journal of cancer. Journal international du cancer. 1993; 55(4): 541-546. PMid:8406979 http://dx.doi.org/10.1002/ijc.2910550404

[12] Haniball J, Sumathi VP, Kindblom LG, et al. Prognostic factors and metastatic patterns in primary myxoid/round-cell liposarcoma. Sarcoma. 2011; 2011:538085. PMid:22190864 http://dx.doi.org/10.1155/2011/538085

[13] Heslin MJ, Gaynor JJ, Newman E, et al. Effect of perioperative blood transfusion on recurrence and survival in 232 primary high-grade extremity sarcoma patients. Annals of surgical oncology. 1994; 1(3): 189-197. PMid:7842288 http://dx.doi.org/10.1007/BF02303523

[14] Jones RL, Fisher C, Al-Muderis O, Judson IR. Differential sensitivity of liposarcoma subtypes to chemotherapy. Eur J Cancer. 2005; 41(18): 2853-2860. PMid:16289617 http://dx.doi.org/10.1016/j.ejca.2005.07.023

[15] Kilpatrick SE, Doyon J, Choong PF, Sim FH, Nascimento AG. The clinicopathologic spectrum of myxoid and round cell liposarcoma. A study of 95 cases. Cancer. 1996; 77(8): 1450-1458. http://dx.doi.org/10.1002/(SICI)1097-0142(19960415)77:8<1450::AID-CNCR5>3.0.CO;2-G

[16] Knight JC, Renwick PJ, Dal Cin P, Van den Berghe H, Fletcher CD. Translocation t(12;16)(q13;p11) in myxoid liposarcoma and round cell liposarcoma: molecular and cytogenetic analysis. Cancer research. 1995; 55(1): 24-27. PMid:7805034

[17] Moreau LC, Turcotte R, Ferguson P, et al. Myxoid \Round Cell Liposarcoma (MRCLS) Revisited: An Analysis of 418 Primarily Managed Cases. Annals of surgical oncology. 2011. PMid:22052112

[18] Newcomer AE, Dylinski D, Rubin BP, et al. Prognosticators in thigh soft tissue sarcomas. Journal of surgical oncology. 2011; 103(1): 85-91. PMid:21165983 http://dx.doi.org/10.1002/jso.21763

[19] Organization WH. International histological classification of tumours. 2nd ed. Berlin: Springer-Verlag; 2002.

[20] Patel SR, Burgess MA, Plager C, Papadopoulos NE, Linke KA, Benjamin RS. Myxoid liposarcoma. Experience with chemotherapy. Cancer. 1994; 74(4): 1265-1269. http://dx.doi.org/10.1002/1097-0142(19940815)74:4<1265::AID-CNCR2820740414>3.0.CO;2-X

[21] Pitson G, Robinson P, Wilke D, et al. Radiation response: an additional unique signature of myxoid liposarcoma. Int J Radiat Oncol Biol Phys. 2004; 60(2): 522-526. PMid:15380587 http://dx.doi.org/10.1016/j.ijrobp.2004.03.009 
[22] Rosenberg SA, Tepper J, Glatstein E, et al. The treatment of soft-tissue sarcomas of the extremities: prospective randomized evaluations of (1) limb-sparing surgery plus radiation therapy compared with amputation and (2) the role of adjuvant chemotherapy. Ann Surg. 1982; 196(3): 305-315. PMid:7114936 http://dx.doi.org/10.1097/00000658-198209000-00009

[23] Smith TA, Easley KA, Goldblum JR. Myxoid/round cell liposarcoma of the extremities. A clinicopathologic study of 29 cases with particular attention to extent of round cell liposarcoma. Am J Surg Pathol. 1996; 20(2): 171-180. PMid:8554106 http://dx.doi.org/10.1097/00000478-199602000-00005

[24] Spillane AJ, Fisher C, Thomas JM. Myxoid liposarcoma--the frequency and the natural history of nonpulmonary soft tissue metastases. Ann Surg Oncol. 1999; 6(4): 389-394. PMid:10379861 http://dx.doi.org/10.1007/s10434-999-0389-5

[25] ten Heuvel SE, Hoekstra HJ, van Ginkel RJ, Bastiaannet E, Suurmeijer AJ. Clinicopathologic prognostic factors in myxoid liposarcoma: a retrospective study of 49 patients with long-term follow-up. Ann Surg Oncol. 2007; 14(1): $222-229$.

PMid:17058128 http://dx.doi.org/10.1245/s10434-006-9043-7 